\title{
The spatial density gradient of galactic cosmic rays and its solar cycle variation observed with the Global Muon Detector Network
}

\author{
Masayoshi Kozai ${ }^{*}$, Kazuoki Munakata ${ }^{1}$, Chihiro Kato ${ }^{1}$, Takao Kuwabara² , John W Bieber ${ }^{2}$, Paul Evenson², \\ Marlos Rockenbach ${ }^{3}$, Alisson Dal Lago ${ }^{4}$, Nelson J Schuch³, Munetoshi Tokumaru ${ }^{5}$, Marcus L Duldig ${ }^{6}$, \\ John E Humble ${ }^{6}$, Ismail Sabbah7, Hala K Al Jassar ${ }^{8}$, Madan M Sharma $^{8}$ and Jozsef Kóta ${ }^{9}$
}

\begin{abstract}
We derive the long-term variation of the three-dimensional (3D) anisotropy of approximately $60 \mathrm{GV}$ galactic cosmic rays (GCRs) from the data observed with the Global Muon Detector Network (GMDN) on an hourly basis and compare it with the variation deduced from a conventional analysis of the data recorded by a single muon detector at Nagoya in Japan. The conventional analysis uses a north-south (NS) component responsive to slightly higher rigidity (approximately $80 \mathrm{GV}$ ) GCRs and an ecliptic component responsive to the same rigidity as the GMDN. In contrast, the GMDN provides all components at the same rigidity simultaneously. It is confirmed that the temporal variations of the 3D anisotropy vectors including the NS component derived from two analyses are fairly consistent with each other as far as the yearly mean value is concerned. We particularly compare the NS anisotropies deduced from two analyses statistically by analyzing the distributions of the NS anisotropy on hourly and daily bases. It is found that the hourly mean NS anisotropy observed by Nagoya shows a larger spread than the daily mean due to the local time-dependent contribution from the ecliptic anisotropy. The NS anisotropy derived from the GMDN, on the other hand, shows similar distribution on both the daily and hourly bases, indicating that the NS anisotropy is successfully observed by the GMDN, free from the contribution of the ecliptic anisotropy. By analyzing the NS anisotropy deduced from neutron monitor (NM) data responding to lower rigidity (approximately 17 GV) GCRs, we qualitatively confirm the rigidity dependence of the NS anisotropy in which the GMDN has an intermediate rigidity response between NMs and Nagoya. From the 3D anisotropy vector (corrected for the solar wind convection and the Compton-Getting effect arising from the Earth's orbital motion around the Sun), we deduce the variation of each modulation parameter, i.e., the radial and latitudinal density gradients and the parallel mean free path for the pitch angle scattering of GCRs in the turbulent interplanetary magnetic field. We show the derived density gradient and mean free path varying with the solar activity and magnetic cycles.
\end{abstract}

Keywords: Diurnal anisotropy; North-south anisotropy; Heliospheric modulation of galactic cosmic rays; Solar cycle variation of the cosmic ray density gradient

\footnotetext{
*Correspondence: 13st303f@shinshu-u.ac.jp

1 Physics Department, Shinshu University, Matsumoto, Nagano 390-8621,

Japan

Full list of author information is available at the end of the article
}

\section{Springer}

(c) 2014 Kozai et al.; licensee Springer. This is an Open Access article distributed under the terms of the Creative Commons

Attribution License (http://creativecommons.org/licenses/by/4.0), which permits unrestricted use, distribution, and reproduction in any medium, provided the original work is properly credited. 


\section{Findings Introduction}

A solar disturbance propagating away from the Sun affects the population of galactic cosmic rays (GCRs) in a number of ways. Using Parker's transport equation (Parker 1965) of GCRs in the heliosphere, we can infer the large-scale spatial gradient of GCR density by measuring the anisotropy of the high-energy GCR intensity. This is influenced by magnetic structures such as interplanetary shocks and magnetic flux ropes in the interplanetary coronal mass ejections (ICMEs). Only a global network of detectors can measure the dynamic variation of the first-order anisotropy accurately and separately from the temporal variation of the GCR density. The Global Muon Detector Network (GMDN) started operation measuring the three-dimensional (3D) anisotropy on an hourly basis with two-hemisphere observations using a pair of muon detectors (MDs) at Nagoya (Japan) and Hobart (Australia) in 1992. In 2001, another small detector at São Martinho (Brazil) was added to the network to fill a gap in directional coverage over the Atlantic and Europe. The current GMDN consisting of four multidirectional muon detectors was completed in 2006 by expanding the São Martinho detector and installing a new detector in Kuwait. Since then, the temporal variations of the anisotropy and density gradient in association with the ICME and corotating interaction regions have been analyzed on an hourly basis using the observations with the GMDN (Rockenbach et al. 2014; Okazaki et al. 2008; Kuwabara et al. 2004; Kuwabara et al. 2009).

Solar cycle variations of the interplanetary magnetic field (IMF) and solar wind parameters also alter the global distribution of GCR density in the heliosphere and cause long-term variations of the 3D anisotropy of the GCR intensity at the Earth. The 'drift model' of cosmic ray transport in the heliosphere, for instance, predicts a bidirectional latitude gradient of the GCR density, pointing in opposite directions on opposite sides of the heliospheric current sheet (HCS) (Kóta and Jokipii 1982). The predicted spatial distribution of the GCR density has a minimum along the HCS in the 'positive' polarity period of the solar polar magnetic field (also referred as $A>0$ epoch), when the IMF directs away from (toward) the Sun in the northern (southern) hemisphere, while the distribution has the local maximum on the HCS in the 'negative' period $(A<0$ epoch) with the opposite field orientation in each hemisphere. The field orientation reverses every 11 years around the period of maximum solar activity. The 3D anisotropy of GCR intensity consists of two components: one lying in the ecliptic plane and the other pointing normal to the ecliptic plane. The ecliptic component can be observed as the solar diurnal anisotropy (the first harmonic vector of the solar diurnal variation) of GCR intensity, while the normal component can be measured as the north-south (NS) anisotropy responsible for the difference between intensities recorded by north- and south-viewing detectors or the sidereal diurnal anisotropy. By analyzing the solar diurnal variation and the NS anisotropy of the GCR intensity recorded by neutron monitors (NMs), Bieber and Chen (1991) and Chen and Bieber (1993) derived the solar cycle variations of 3D anisotropy and modulation parameters on a yearly basis. On the other hand, Munakata et al. (2014) derived the long-term variation of the $3 \mathrm{D}$ anisotropy from the longterm record of the GCR intensity observed with a single multidirectional MD at Nagoya in Japan. By comparing the anisotropy derived from the MD data with that from the NM data, they examined the rigidity dependence of the anisotropy and its solar cycle variation.

Accurate observation of the NS anisotropy normal to the ecliptic plane is also crucial for obtaining a reliable 3D anisotropy. This component has been derived from NM and MD data in two different ways. Chen and Bieber (1993) derived this component anisotropy from the difference between count rates in a pair of NMs which are located near the north and south geomagnetic poles and observing intensities of GCRs arriving from the north and south pole orientations, respectively. The NS anisotropy derived in this way is very sensitive to the stability of operations of two independent detectors and can be easily affected by unexpected changes of instrumental and/or environmental origins. Due to the $23.4^{\circ}$ inclination of Earth's rotation axis from the ecliptic normal, the NS anisotropy normal to the ecliptic plane can be also observed as a diurnal variation of count rate in sidereal time with the maximum phase at approximately $06: 00$ or approximately 18:00 local sidereal time (Swinson 1969). A possible drawback of deriving the NS anisotropy from the sidereal diurnal variation is that the expected amplitude of the sidereal diurnal variation is roughly ten times smaller than that of the solar diurnal variation. The small signal in sidereal time can be easily influenced by the solar diurnal anisotropy changing during a year. Another difficulty is that one can obtain only the yearly mean anisotropy, because the influence from the solar diurnal variation, even if it is stationary throughout a year, can be canceled in sidereal time only when the diurnal variation is averaged over at least 1 year. This makes it difficult to deduce a reliable error of the yearly mean anisotropy. Mori and Nagashima (1979) proposed another way to derive the NS anisotropy from the 'GG-component' of a multidirectional MD at Nagoya in Japan. The GG-component is a difference combination between intensities recorded in the north- and south-viewing directional channels corresponding to $56^{\circ}$ north and $14^{\circ}$ south asymptotic latitudes in free space at their median rigidity, approximately $80 \mathrm{GV}$, designed to measure the NS anisotropy free from atmospheric temperature effect (Nagashima et al. 
1972). The NS anisotropy depends on the polarity of the magnetic field. Based on this fact, Laurenza et al. (2003) showed that the GG-component can be used for deriving reliable sector polarity of the IMF which is defined as away (toward) when the IMF directs away from (toward) the Sun. By using a global network of four multidirectional MDs which is able to observe the NS anisotropy on an hourly basis, Okazaki et al. (2008) reported for the first time that the NS anisotropy deduced from the GGcomponent is consistent with the anisotropy observed with the global network for a year during the solar activity minimum period.

Analyses of the diurnal variation observed with a single detector, however, can give a correct anisotropy only when the anisotropy is stationary at least over 1 day and may not work if the anisotropy changes dynamically within a day. The GG-component also needs to be averaged over 1 day to cancel the influence of the ecliptic components which have components parallel to the rotation axis of the Earth and contribute to the NS difference measured by the GG-component. Additionally, the directional channels of the Nagoya MD have an angular distribution biased toward the northern hemisphere, while the GMDN has a global angular distribution. It is important, therefore, to examine whether the long-term variation of the $3 \mathrm{D}$ anisotropy derived from the conventional analysis of the observed diurnal variation and the GG-component is consistent with the anisotropy observed by the GMDN which is capable of accurately measuring anisotropy with better time resolution. In this paper, we analyze the 3D anisotropy observed with the GMDN over 22 years between 1992 and 2013 and compare it with the anisotropy observed with the Nagoya multidirectional MD, especially focusing on the NS anisotropy for which the GGcomponent has been the only reliable measurement at the 50 to $100 \mathrm{GV}$ region. Based on the difference of the response rigidities between the GMDN (approximately 60 GV) and the GG-component (approximately $80 \mathrm{GV}$ ), we also discuss the rigidity dependence of the NS anisotropy.

\section{Data analysis}

We analyze the pressure-corrected hourly count rate $I_{i, j}(t)$ of recorded muons in the $j$ th directional channel of the $i$ th detector in the GMDN at universal time $t$ and derive three components $\left(\xi_{x}^{\mathrm{GEO}}(t), \xi_{y}^{\mathrm{GEO}}(t), \xi_{z}^{\mathrm{GEO}}(t)\right)$ of the firstorder anisotropy in the geographic (GEO) coordinate system by best fitting the following model function to $I_{i, j}(t)$.

$$
\begin{aligned}
f_{i, j}^{f i t}(t)=I_{i, j}^{0}(t) & +\xi_{x}^{\mathrm{GEO}}(t)\left(c_{1 i, j}^{1} \cos \omega t_{i}-s_{1 i, j}^{1} \sin \omega t_{i}\right) \\
& +\xi_{y}^{\mathrm{GEO}}(t)\left(s_{1 i, j}^{1} \cos \omega t_{i}+c_{1 i, j}^{1} \sin \omega t_{i}\right) \\
& +\xi_{z}^{\mathrm{GEO}}(t) c_{1 i, j}^{0}
\end{aligned}
$$

where $I_{i, j}^{0}(t)$ is a parameter representing the contributions from the omnidirectional intensity and the atmospheric temperature effect; $t_{i}$ is the local time at the $i$ th detector; $c_{1 i, j}^{1}, s_{1 i, j}^{1}$, and $c_{1 i, j}^{0}$ are the coupling coefficients; and $\omega=\pi / 12$. The coupling coefficients are calculated by integrating the response function of atmospheric muons to the primary cosmic rays (Murakami et al. 1979) for primary rigidity, detective solid angle, and detection area with weights of the asymptotic orbit by assuming a rigidity-independent anisotropy with the upper limiting rigidity set at $10^{5} \mathrm{GV}$, far above the maximum rigidity of the response.

In deriving the anisotropy vector $\xi$, we additionally apply an analysis method developed to remove the influence of atmospheric temperature variations from the derived anisotropy (see Okazaki et al. 2008). Elimination of the temperature effect from the MD data is of particular importance in analyzing the long-term temporal variation of $\xi$. The deduced anisotropy is averaged over each IMF sector in every month designated as away (toward) if the daily polarity of the Stanford mean magnetic field of the Sun (Wilcox Solar Observatory), shifted 5 days later for a rough correction for the solar wind transit time between the Sun and the Earth, is positive (negative).

We also derive the anisotropy from observations by a single multidirectional MD at Nagoya (hereafter Nagoya $\mathrm{MD}$ ) which is a component detector of the GMDN. By using the coupling coefficients, we deduce the equatorial component $\left(\xi_{x}^{\mathrm{GEO}}, \xi_{y}^{\mathrm{GEO}}\right)$ of $\xi$ from the mean diurnal variation of the hourly counting rate in each IMF sector in every month. On the other hand, we derive the normal component to the equatorial plane, $\xi_{z}^{\mathrm{GEO}}$, from the GG-component averaged over each IMF sector by using the coupling coefficient in every month (Munakata et al. 2014). The GG-component is a difference combination between intensities recorded in the north- and south-viewing channels and has long been used as a good measure of the NS anisotropy (Mori and Nagashima 1979; Nagashima et al. 1972; Laurenza et al. 2003).

The anisotropy vector $\left(\xi_{x}^{\mathrm{GEO}}, \xi_{y}^{\mathrm{GEO}}, \xi_{z}^{\mathrm{GEO}}\right)$ in three dimensions derived from the GMDN and Nagoya data is transformed to the geocentric solar ecliptic (GSE) coordinate system, in which the $z$-component corresponds to the NS component normal to the ecliptic plane, and corrected for the solar wind convection anisotropy using the solar wind velocity in the 'omnitape' (King and Papitashvili 2005; NASA 2014) data by the Space Physics Data Facility at the Goddard Space Flight Center and for the ComptonGetting anisotropy arising from the Earth's $30 \mathrm{~km} / \mathrm{s}$ orbital motion around the Sun. In the corrections, we set the power law index of the GCR energy spectrum to be -2.7 . We then obtain the ecliptic plane component of the anisotropy consisting of components parallel $\left(\xi_{\|}\right)$and 
perpendicular $\left(\xi_{\perp}\right)$ to the IMF as obtained from the omnitape data and NS anisotropy $\left(\xi_{z}\right)$ normal to the plane in each IMF sector in every month. We finally obtain the monthly mean three components of the anisotropy in the solar wind frame as

$$
\begin{aligned}
& \xi_{\|}=\left(\xi_{\|}^{T}+\xi_{\|}^{A}\right) / 2 \\
& \xi_{\perp}=\left(\xi_{\perp}^{T}+\xi_{\perp}^{A}\right) / 2 \\
& \xi_{z}=\left(\xi_{z}^{T}-\xi_{z}^{A}\right) / 2
\end{aligned}
$$

where $\xi_{\|}^{T}\left(\xi_{\|}^{A}\right)$ and $\xi_{\perp}^{T}\left(\xi_{\perp}^{A}\right)$ are parallel and perpendicular components in the ecliptic plane averaged over the toward (away) sector, while $\xi_{z}^{T}\left(\xi_{z}^{A}\right)$ is the NS anisotropy in the toward (away) sector. We assume that the anisotropy vector, when averaged over 1 month exceeding a solar rotation period, is symmetrical with respect to the HCS which is regarded to coincide with the solar equatorial plane on the average. Because of this assumption, the NS anisotropy is directed oppositely, with the same magnitude, above and below the HCS as defined in Equation 2c.

\section{Solar cycle variation of the 3D anisotropy}

Figure 1a,b,c shows the temporal variations of the yearly mean $\xi_{\|}, \xi_{\perp}$, and $\xi_{z}$ as defined in Equation 2c, respectively. Each panel shows that the temporal variations of the anisotropy components derived from the GMDN (solid circle) and Nagoya (open circle) data are fairly consistent with each other as far as the year-to-year variation is concerned. We can see that the solar cycle variation of $\xi_{\|}$has two components. One is a 22-year variation resulting in a slightly larger $\xi_{\|}$in $A<0$ epoch (2001 to 2011) than in $A>0$ epoch (1992 to 1998) as reported by Chen and Bieber (1993). The other is a variation correlated with $\cos \psi$, shown with $\xi_{\|}$by open squares in Figure 1a, where $\psi$ is the IMF spiral angle derived from omnitape data. $\xi_{z}$ deduced from the GMDN (solid circles), on the other hand, shows an 11-year cycle with minima in 1998 and 2007 around the solar activity minima, while $\xi_{\perp}$ shows no solar cycle variation.

\section{Comparison between the NS anisotropies observed with the GMDN and the GG-component}

We now focus on the NS anisotropy which cannot be detected by a single-directional channel separately from GCR density variations. Figure 2 shows histograms of hourly (a and b) and daily (c and d) mean $\xi_{z}^{\mathrm{GEO}}$ observed by the GG-component ( $\mathrm{a}$ and c) and GMDN (b and d) in 2006 to 2013, which are classified according to the IMF sectors designated as toward (blue histograms) if $B_{x}>B_{y}$ and away (red histograms) if $B_{x}<B_{y}$ by using the GSE- $x, y$ components $\left(B_{x}, B_{y}\right)$ of the IMF vector in the omnitape data. The blue and red vertical dashed lines represent averages of the blue and red histograms, respectively. We define ' $T$ / $A$ separation' following Okazaki et al. (2008) as

$$
(T-A) / \sqrt{\sigma_{T} \sigma_{A}}
$$

where $T(A)$ and $\sigma_{T}\left(\sigma_{A}\right)$ are the average and standard errors of each histogram in the toward (away) sector,

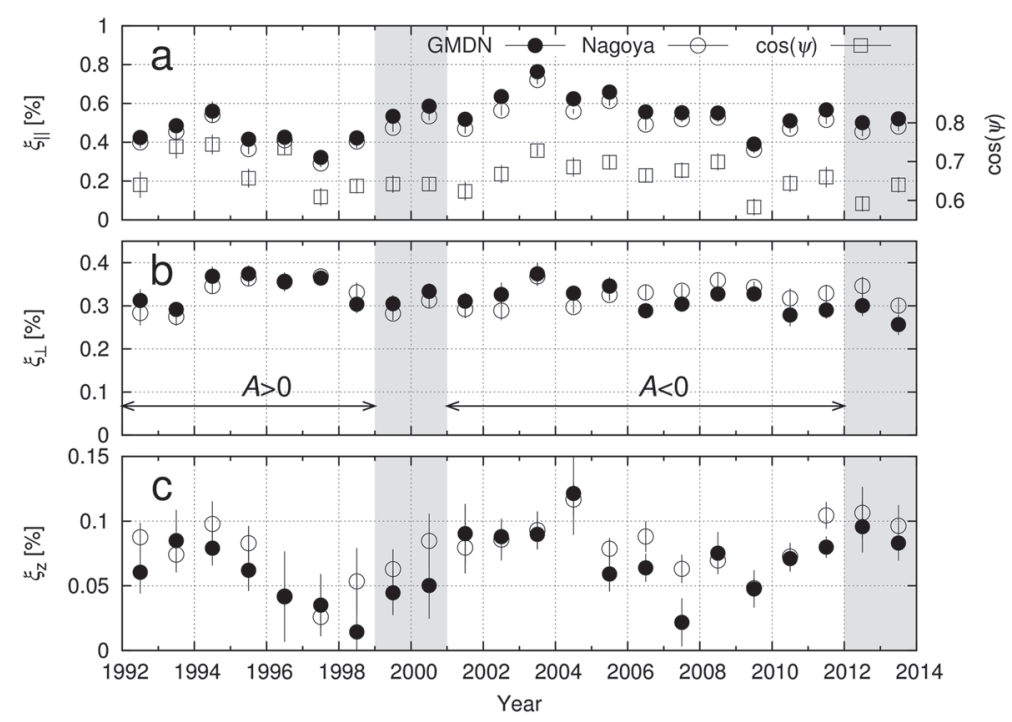

Figure 1 Long-term variations of three components of the anisotropy vector in the solar wind frame. Each panel displays the yearly mean (a) $\xi_{\|}$(on the left vertical axis), (b) $\xi_{\perp}$, and (c) $\xi_{z}$ as defined in Equation 2c, each as a function of year on the horizontal axis. Solid and open circles in each panel represent anisotropies derived from the GMDN and Nagoya data, respectively, while open squares in (a) display $\cos \psi$ on the right vertical axis. In each panel, yearly mean value and its error are deduced from the average and dispersion of monthly mean values. Gray vertical stripes indicate periods when the polarity reversal of the solar polar magnetic field (referred as $A>0$ or $A<0$ in (b)) is in progress. 


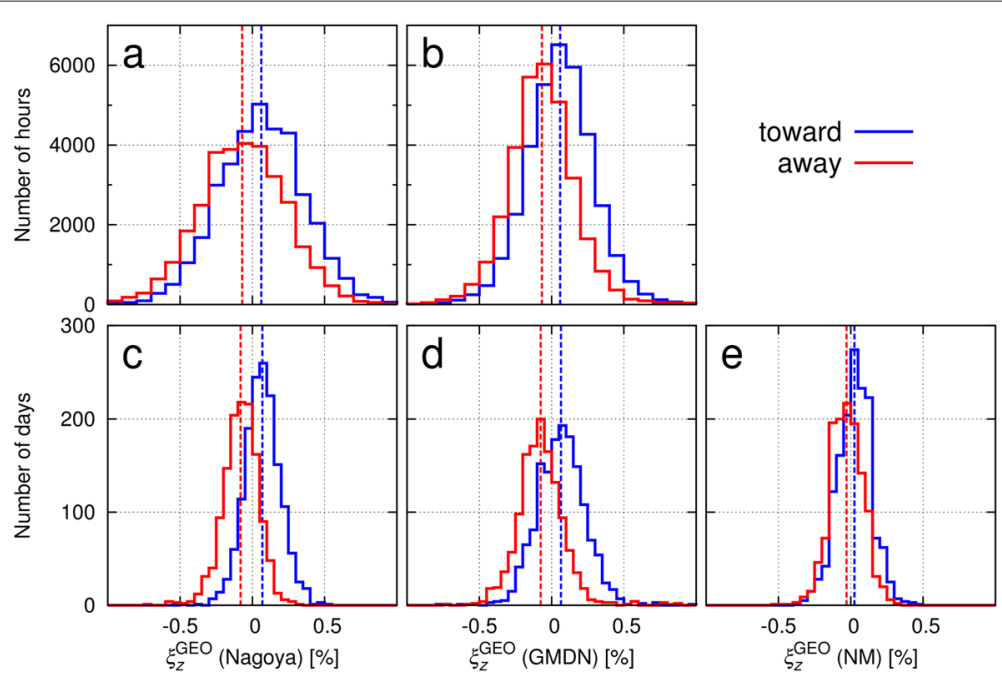

Figure 2 Histograms of the NS anisotropy. Each panel displays the histograms of $\xi_{z}^{\mathrm{GEO}}$ on (a, b) hourly and (c, $\left.\mathbf{d}, \mathbf{e}\right)$ daily bases derived from the (a, c) Nagoya GG-component, (b, d) GMDN, and (e) NM (Thule-McMurdo) data in 2006 to 2013. Blue and red histograms in each panel represent distributions of $\xi_{z}^{\mathrm{GEO}}$ in toward and away IMF sectors, respectively, while blue and red vertical dashed lines represent averages of the blue and red histograms, respectively.

respectively. Table 1 lists $T-A, \sqrt{\sigma_{T} \sigma_{A}}, T / A$ separation, and 'success rate' (Mori and Nagashima 1979; Laurenza et al. 2003). The success rate is a ratio of the number of hours (days) when the sign of the observed $\xi_{z}^{\mathrm{GEO}}$ is positive (negative) in the toward (away) IMF sector to the total number of hours (days) and is introduced as a parameter indicating to what extent we can infer the IMF sector polarity from the sign of the observed $\xi_{z}$. Although we use the success rate together with $T / A$ separation for the following comparison, it is noted that a low success rate does not necessarily imply anything wrong in the observed $\xi_{z}$. The IMF sector polarity sensed by high-energy GCRs should be regarded as the polarity averaged over a spatial scale comparable to the Larmor radii of GCRs which span approximately $0.1 \mathrm{AU}$. It is natural to expect that the IMF polarity averaged over such a large scale does not always

Table $1 T-A, \sqrt{\sigma_{T} \sigma_{A}}, T / A$ separation, and success rate

\begin{tabular}{lcccc}
\hline & & Nagoya & GMDN & NMs \\
\hline$T-A(\%)$ & Daily & 0.1504 & 0.1398 & 0.0546 \\
& Hourly & 0.1324 & 0.1258 & - \\
$\sqrt{\sigma_{T} \sigma_{A}}(\%)$ & Daily & 0.0033 & 0.0044 & 0.0031 \\
& Hourly & 0.0016 & 0.0013 & - \\
$T / A$ separation & Daily & 46.2 & 31.5 & 17.8 \\
& Hourly & 81.2 & 96.6 & - \\
Success rate (\%) & Daily & 73.5 & 68.7 & 59.6 \\
& Hourly & 58.2 & 62.0 & - \\
\hline
\end{tabular}

Difference $(T-A)$ between average $\xi_{z}^{\text {GEO }}$ in toward $(T)$ and away $(A)$ IMF sectors, geometric mean $\left(\sqrt{\sigma_{T} \sigma_{A}}\right)$ of the standard errors of $\xi_{Z}^{\mathrm{GEO}} \mathrm{s}$ in $T$ and $A$ sectors, 'T/A separation' $\left(=(T-A) / \sqrt{\sigma_{T} \sigma_{A}}\right)$, and 'success rate' (see text) derived from Nagoya, GMDN, and NM (Thule-McMurdo) data in 2006 to 2013 on daily and hourly bases. follow the single-point measurement of the polarity by a satellite. In Table 1 , it is seen that the daily mean $\xi_{z}^{\mathrm{GEO}}$ by the GMDN shows smaller $T / A$ separation and success rate than $\xi_{z}^{\mathrm{GEO}}$ deduced from the GG-component, while the hourly $\xi_{z}^{\mathrm{GEO}}$ by GMDN has a larger $T / A$ separation and success rate than the GG-component which has significantly larger dispersion (Figure 2a), partly due to the contribution from diurnal anisotropy as suggested by Okazaki et al. (2008) from their analysis of 1-year data between March 2006 and March 2007.

We also examine the rigidity dependence of the NS anisotropy by analyzing NM data from 2006 to 2013. NMs have median responses to approximately 17 GV GCRs, while the GMDN and GG-component have median responses to approximately $60 \mathrm{GV}$ and approximately $80 \mathrm{GV}$, respectively. Chen and Bieber (1993) derived the NS anisotropy $\xi_{z}$ in Equation 2c from the ratio $(R)$ of the daily mean counting rate recorded by the Thule NM to that recorded by the McMurdo NM as

$$
\xi_{z}=\frac{b}{2} \frac{R^{T}-R^{A}}{R^{T}+R^{A}}
$$

where $R^{T}\left(R^{A}\right)$ is the $R$ averaged over toward (away) sectors in every month and $b$ is a constant calculated from coupling coefficients. We define the daily mean NS anisotropy by NMs as

$$
\xi_{z}^{\mathrm{GEO}}=\frac{c}{2} \frac{R}{R^{T}+R^{A}}
$$

where $c$ is a coupling coefficient calculated on the same assumption as adopted in our analysis of the GMDN and Nagoya MD data. The $T / A$ separation and success rate of 
this $\xi_{z}^{\mathrm{GEO}}$ represents those parameters for approximately 17 GV GCRs. The result of this analysis is presented in Figure $2 \mathrm{e}$ and Table 1 . It is seen that the $T / A$ separation of the NS anisotropy by NMs is significantly smaller mainly due to the small $T-A$, i.e., the NS anisotropy is significantly smaller than that obtained from the GMDN and GG-component. The NS anisotropy is smallest in NM data at approximately $17 \mathrm{GV}$ and largest in the GGcomponent at approximately $80 \mathrm{GV}$, with the anisotropy in the GMDN at approximately $60 \mathrm{GV}$ in between, suggesting that the NS anisotropy increases with increasing rigidity (Munakata et al. 2014).

\section{Solar cycle variation of modulation parameters}

Following the analyses by Chen and Bieber (1993), we derive modulation parameters, i.e., the density gradient and the mean free path of the pitch angle scattering. By assuming that the longitudinal gradient is zero in our analysis based on the anisotropy averaged over 1 month which is longer than the solar rotation period, $\xi_{\|}, \xi_{\perp}$, and $\xi_{z}$ obtained in Equations 2a, 2b, and 2c are related with the modulation parameters as

$$
\begin{aligned}
\xi_{\|} & =\lambda_{\|} G_{r} \cos \psi \\
\xi_{\perp} & =\lambda_{\perp} G_{r} \sin \psi-R_{L} G_{z} \\
\xi_{z} & =R_{L} G_{r} \sin \psi+\lambda_{\perp} G_{z}
\end{aligned}
$$

where $R_{L}$ is the Larmor radius of GCRs in the IMF and $G_{z}, G_{r}, \lambda_{\|}$, and $\lambda_{\perp}$ are the latitudinal and radial density gradients and the mean free paths of the pitch angle scattering parallel and perpendicular to the IMF.
From Equations 5a, 5b, and 5c, we deduce the modulation parameters as

$$
\begin{aligned}
& G_{z}=\left(\alpha \xi_{\|} \tan \psi-\xi_{\perp}\right) / R_{L} \\
& G_{r}=\left\{\xi_{z}+\sqrt{\xi_{z}^{2}+4 \alpha \xi_{\|} \tan \psi\left(\xi_{\perp}-\alpha \xi_{\|} \tan \psi\right)}\right\} /\left(2 R_{L} \sin \psi\right) \\
& \lambda_{\|}=\xi_{\|} /\left(G_{r} \cos \psi\right)
\end{aligned}
$$

where $\alpha=\lambda_{\perp} / \lambda_{\|}$, assumed to be 0.01 and constant as adopted by Chen and Bieber (1993). $G_{z}$ is converted to the bidirectional latitudinal gradient as

$$
G_{|z|}=-\operatorname{sgn}(A) G_{z}
$$

where $A$ represents the polarity of the solar dipole magnetic moment and

$$
\begin{aligned}
\operatorname{sgn}(A) & =+1, \text { for } A>0 \text { epoch, } \\
& =-1, \text { for } A<0 \text { epoch. }
\end{aligned}
$$

Figure 3a,b,c displays temporal variations of modulation parameters $G_{|z|}, G_{r}$, and $\lambda_{\|}$, respectively, obtained from the GMDN (solid circle) and Nagoya (open circle) data. The variations with 22-year and 11-year solar cycles are clearly seen in this figure. First, $G_{|z|}$ is positive in $A>0$ epoch indicating the local minimum of the density on the HCS, while it is negative in $A<0$ epoch indicating the maximum in accord with the prediction of the drift model by Kóta and Jokipii (1983). Second, significant 11-year variations are seen in both $G_{r}$ and $\lambda_{\|}$which change in a clear anti-correlation.

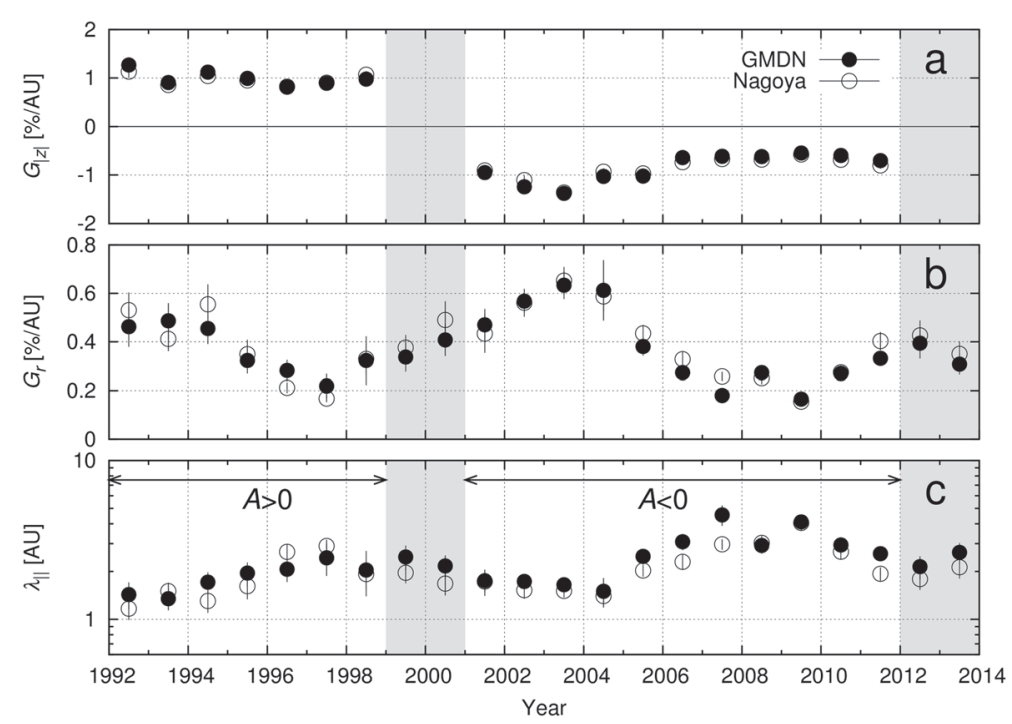

Figure 3 Long-term variations of modulation parameters derived from the 3D anisotropy in the solar wind frame. Each panel displays the yearly mean (a) $G_{|z|}$, (b) $G_{r}$, and (c) $\lambda_{\|}$as a function of year. Solid and open circles in each panel represent parameters derived from the GMDN and Nagoya data, respectively. In each panel, yearly mean value and its error are deduced from the average and dispersion of monthly mean values. Gray vertical stripes indicate periods when the polarity reversal of the solar polar magnetic field (referred as $A>0$ or $A<0$ in (c)) is in progress. 


\section{Summary and discussions}

We analyzed the 3D anisotropy of GCR intensity observed by the GMDN and Nagoya MD in 1992 to 2013. Our analysis of the GMDN data gives the anisotropy on an hourly basis with better time resolution than the traditional analyses of the diurnal and NS anisotropies observed by a single detector such as the Nagoya MD. We confirmed that the 3D anisotropy and the modulation parameters derived from the GMDN and Nagoya MD data are fairly consistent with each other as far as the yearly mean value is concerned. This fact is important particularly for the NS anisotropy derived from the GMDN data, because the GGcomponent has been the only reliable reference to the NS anisotropy in the rigidity region between 50 and $100 \mathrm{GV}$.

By analyzing the distribution of the NS anisotropy separately in toward and away IMF sectors, we compared the $T / A$ separations and success rates deduced from the GMDN and Nagoya MD data on hourly and daily bases. It is confirmed that the daily mean NS anisotropy observed by the GG-component shows slightly better $T / A$ separation and success rate than the daily mean anisotropy by the GMDN, while the hourly mean NS anisotropy by the GG-component shows a large spread due to the local time-dependent contribution from the ecliptic anisotropy. The NS anisotropy by the GMDN, on the other hand, shows similar success rate on both daily and hourly bases, indicating that the NS anisotropy is successfully observed by the GMDN, free from the contribution of the ecliptic anisotropy.

In addition to the better time resolution, the new analysis method developed by Okazaki et al. (2008) for the GMDN data also has an advantage of providing the 3D anisotropy, including the NS component, from a single best-fit calculation for intensities recorded by four detectors. In contrast, the conventional method using a single MD requires the derivation of the NS anisotropy from the north- and south-viewing channels, separately from the derivation of the diurnal anisotropy using all directional channels.

By comparing the NS anisotropy derived from NM data with those from the GMDN and GG-component data, we find that the NS anisotropy increases with increasing rigidity and the difference between $T / A$ separations and success rates of the GMDN and GG-component data is partly due to the rigidity dependence. Yasue (1980) analyzed the GG-component together with the sidereal diurnal variation observed by MDs and NMs and derived the power law-type rigidity spectrum of the average NS anisotropy with a positive power law index of approximately 0.3 . By analyzing long-term variations of the 3D anisotropies observed with Nagoya MD and NMs on yearly basis, Munakata et al. (2014) confirmed that the perpendicular component including the NS anisotropy increases with GCR rigidity. If these are the case, the magnitude of the NS anisotropy increases with rigidity and the $T / A$ separation and success rate will also increase if the dispersion remains similarly independent of rigidity. This is in agreement with our results in Table 1, showing that $T-A$ increases with the rigidity while $\sqrt{\sigma_{T} \sigma_{A}}$ is almost constant on a daily basis. Three GCR observations responsible for different rigidities, GG-component (approximately $80 \mathrm{GV}$ ), GMDN (approximately $60 \mathrm{GV}$ ), and NMs (approximately $17 \mathrm{GV}$ ) are capable of observing the NS anisotropy on daily basis, and their crosscalibration allows us to obtain the information about the rigidity dependence of the NS anisotropy.

We confirmed that the solar cycle variations of the yearly mean solar modulation parameters derived from the GMDN and Nagoya data are consistent with each other. The bidirectional latitudinal gradient $G_{|z|}$ shows a clear 22-year variation being positive (negative) in $A>0(A<0)$ epochs indicating the local minimum (maximum) of the GCR density on the HCS, in accord with the prediction of the drift model (Kóta and Jokipii 1983). On the other hand, significant 11-year solar cycle variations are seen in $G_{r}$ and $\lambda_{\|}$, respectively. The ecliptic component of the anisotropy $\xi_{\|}$parallel to the IMF shows a 22-year variation being slightly larger in $A<0$ epoch (2001 to 2011) than in $A>0$ epoch (1992 to 1998) as reported by Chen and Bieber (1993). This variation of $\xi_{\|}$is responsible for the well-known 22-year variation of the phase of the diurnal variation (Thambyahpillai and Elliot 1953). We find that the variation of $\xi_{\|}$also shows a correlation with the $\cos \psi$

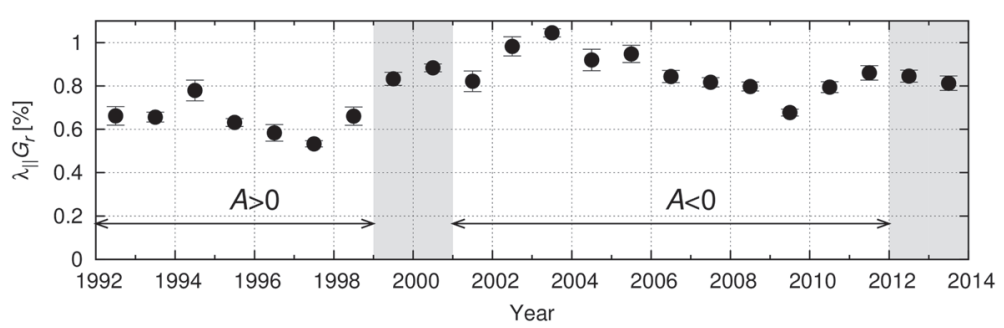

Figure 4 Long-term variation of $\lambda_{\|} \boldsymbol{G}_{\boldsymbol{r}}$ derived from the GMDN data. Yearly mean value and its error are deduced from the average and dispersion of monthly mean values. Gray vertical stripes indicate periods when the polarity reversal of the solar polar magnetic field (referred as $A>0$ or $A<0)$ is in progress. 
which is governed by the solar wind velocity. This is reasonable because $\xi_{\|}$is proportional to $\cos \psi$ as given in Equation $5 \mathrm{a}$. Figure 4 shows yearly variation of $\xi_{\|} / \cos \psi$, i.e., $\lambda_{\|} G_{r}$ by the GMDN. In this figure, the 22-year variation is seen more clearly than in Figure 1 showing $\xi_{\|}$(Chen and Bieber 1993). For an accurate analysis of the solar cycle variation of the anisotropy, therefore, it is necessary to correct the observed anisotropy for $\cos \psi$ and the solar wind velocity which varies without any clear 11-year or 22-year periodicities.

The 22-year variation of $\lambda_{\|} G_{r}$ seems mainly due to the variation of $\lambda_{\|}$in Figure 3 which is larger in $A<0$ epoch (2001 to 2011) than in $A>0$ epoch (1992 to 1998). However, the solar magnetic field was unusually weak around this last solar minimum (2009), which resulted in a recordhigh GCR flux (Mewaldt et al. 2010). The larger mean free path is more likely the result of the weaker solar minimum than a polarity issue.

\section{Competing interests}

The authors declare that they have no competing interests.

\section{Authors' contributions}

MK, the corresponding author of this paper, made all analyses presented in this paper. KM evaluated the data and discussed the analysis results in this paper. CK made the GMDN data available for this paper. TK made the GMDN data and the neutron monitor data available for this paper. JWB and PE made the neutron monitor data available for this paper. MR kept the São Martinho muon detector in operation. ADL hosted the São Martinho muon detector, assisted with its installation, and performed its general maintenance. NJS contributed in establishing the observation with the São Martinho muon detector and kept it in operation. MT kept the Nagoya muon detector in operation. MLD and JEH hosted the Hobart muon detector, assisted with its installation, performed its general maintenance, discussed the results, and input into the English grammar of this paper. IS established the observation with the Kuwait muon detector. HKAJ and MMS kept the Kuwait muon detector in operation. JK discussed analysis results and worked for improving the English in this paper. All authors read and approved the final manuscript.

\section{Acknowledgements}

This work is supported in part by the joint research programs of the SolarTerrestrial Environment Laboratory (STEL), Nagoya University and the Institute for Cosmic Ray Research (ICRR), University of Tokyo. The observations with the Nagoya multidirectional muon detector are maintained by Nagoya University. CNPq, CAPES, INPE and UFSM support upgrade and maintenance of the São Martinho muon detector. The Bartol Research Institute neutron monitor program, which operates Thule and McMurdo neutron monitors, is supported by National Science Foundation grant ATM-0000315. Wilcox Solar Observatory data used in this study was obtained via the website http://wso.stanford.edu at 2013:06:24\$ $¥$ 22:12:55 PDT courtesy of J.T. Hoeksema. The Wilcox Solar Observatory is currently supported by NASA. JK thanks STEL and Shinshu University for the hospitality during his stay as a visiting professor of STEL.

\section{Author details}

${ }^{1}$ Physics Department, Shinshu University, Matsumoto, Nagano 390-8621, Japan. ${ }^{2}$ Bartol Research Institute, Department of Physics and Astronomy, University of Delaware, Newark, DE 19716, USA. ${ }^{3}$ Southern Regional Space Research Center (CRS/INPE), P.O. Box 5021, 97110-970, Santa Maria RS, Brazil. ${ }^{4}$ National Institute for Space Research (INPE), 12227-010, São José dos Campos SP, Brazil. ${ }^{5}$ Solar Terrestrial Environment Laboratory, Nagoya University, Nagoya, Aichi 464-8601, Japan. ${ }^{6}$ School of Physical Sciences, University of Tasmania, Hobart, Tasmania 7001, Australia. ${ }^{7}$ Department of Natural Sciences, College of Health Sciences, Public Authority for Applied Education and Training, Kuwait City 72853, Kuwait. ${ }^{8}$ Physics Department, Kuwait University, Kuwait City 13060 , Kuwait. ${ }^{9}$ Lunar and Planetary Laboratory, University of Arizona, Tucson, AZ 85721, USA.
Received: 31 March 2014 Accepted: 29 October 2014

Published online: 14 November 2014

\section{References}

Bieber JW, Chen J (1991) Cosmic-ray diurnal anisotropy, 1936-1988: implications for drift and modulation theories. Astrophys J 372:301-313

Chen J, Bieber JW (1993) Cosmic-ray anisotropies and gradients in three dimensions. Astrophys J 405:375-389

King JH, Papitashvili NE (2005) Solar wind spatial scales in and comparisons of hourly wind and ace plasma and magnetic field data. J Geophys Res 110 : A02104:1-8

Kóta J, Jokipii JR (1982) Cosmic rays near the heliospheric current sheet. Geopys Res Lett 9:656-659

Kóta J, Jokipii JR (1983) Effects of drift on the transport of cosmic rays. VI. A three-dimensional model including diffusion. Astrophys J 265:573-581

Kuwabara T, Munakata K, Yasue S, Kato C, Akahane S, Koyama M, Bieber JW, Evenson P, Pyle R, Fujii Z, Tokumaru M, Kojima M, Marubashi K, Duldig ML, Humble JE, Silva MR, Trivedi NB, Gonzalez WD, Schuch NJ (2004) Geometry of an interplanetary CME on October 29, 2003 deduced from cosmic rays. Geophys Res Lett 31: L19803:1-5

Kuwabara T, Bieber JW, Evenson P, Munakata K, Yasue S, Kato C, Fushishita A, Tokumaru M, Duldig ML, Humble JE, Silva MR, Lago AD, Schuch NJ (2009) Determination of interplanetary coronal mass ejection geometry and orientation from ground-based observations of galactic cosmic rays. J Geophys Res 114: A05109:1-10

Laurenza M, Storini M, Moreno G, Fujii Z (2003) Interplanetary magnetic field polarities inferred from the north-south cosmic ray anisotropy. J Geophys Res 108:1069-1075

Mewaldt RA, Davis AJ, Lave KA, Leske RA, Stone EC, Wiedenbeck ME, Binns WR, Christian ER, Cummings AC, de Nolfo GA, Israel MH, Labrador AW, von Rosenvinge TT (2010) Record-setting cosmic-ray intensities in 2009 and 2010. Astrophys J Lett 723:1-6

Mori S, Nagashima K (1979) Inference of sector polarity of the interplanetary magnetic field from the cosmic ray north-south asymmetry. Planet Space Sci 27:39-46

Murakami K, Nagashima K, Sagisaka S, Mishima Y, Inoue A (1979) Response functions for cosmic-ray muons at various depths underground. IL Nuovo Cimento 2C:635-651

Munakata K, Kozai M, Kato C (2014) Long term variation of the solar diurnal anisotropy of galactic cosmic rays observed with the Nagoya multi-directional muon detector. Astrophys J 791: 22:1-16

Nagashima K, Fujimoto K, Fujii Z, Ueno H, Kondo I (1972) Three-dimensional cosmic ray anisotropy in interplanetary space. Rep Ionos Space Res J.pn 26:31-68

NASA (2014) The "omnitape" data accessed on February 10, 2014 http://omniweb.gsfc.nasa.gov/

Okazaki Y, Fushishita A, Narumi T, Kato C, Yasue S, Kuwabara T, Bieber JW, Evenson P, Silva MRD, Lago AD, Schuch NJ, Fujii Z, Duldig ML, Humble JE, Sabbah I, Kóta J, Munakata K (2008) Drift effects and the cosmic ray density gradient in a solar rotation period: first observation with the Global Muon Detector Network (GMDN). Astrophys J 681:693-707

Parker EN (1965) The passage of energetic charged particles through interplanetary space. Planet Space Sci 13:9-49

Rockenbach M, Lago AD, Schuch NJ, Munakata K, Kuwabara T, Oliveira AG, Echer E, Braga CR, Mendonca RRS, Kato C, Kozai M, Tokumaru M, Bieber JW, Evenson P, Duldig ML, Humble JE, Jassar HKA, Sharma MM, Sabbah I (2014) Global muon detector network used for space weather applications. Space Sci Rev 182:1-18

Swinson DB (1969) "Sidereal" cosmic-ray diurnal variations. J Geophys Res 74:5591-5598

Thambyahpillai T, Elliot H (1953) World-wide changes in the phase of the cosmic-ray solar daily variation. Nature 171:918-920

Yasue S (1980) North-south anisotropy and radial density gradient of galactic cosmic rays. J Geomag Geoelectr 32:617-635

doi:10.1186/s40623-014-0151-5

Cite this article as: Kozai et al:: The spatial density gradient of galactic cosmic rays and its solar cycle variation observed with the Global Muon Detector Network. Earth, Planets and Space 2014 66:151. 